Bull. Austral. Math. Soc.

30D35, 30D30

Vol. 75 (2007) [459-468]

\title{
ON A NEW SINGULAR DIRECTION OF ALGEBROID FUNCTIONS
}

\author{
Songmin Wang and Zongsheng GaO
}

In this paper, we prove that for an algebroid function $w(z)$ with finite lower order, satisfying $\limsup _{r \rightarrow \infty}\left(T(r, w) /(\log r)^{2}\right)=\infty$, there exists a $T$ direction dealing with multiple values.

\section{INTRODUCTION AND MAIN RESULTS}

The $\nu$-valued algebroid function $w=w(z)(z \in \mathrm{C})$ is defined by the irreducible equation(see $[5,6])$

$$
A_{\nu}(z) w^{\nu}+A_{\nu-1}(z) w^{\nu-1}+\ldots+A_{0}(z)=0,
$$

where $A_{\nu}(z), \ldots, A_{0}(z)$ are all entire functions without any common zeros. In particular, $w(z)$ is a meromorphic function when $\nu=1$. In 1930s, Valiron [13], Ullrich [12] and Seleberg $[10,11]$ generalised the value distribution theory of meromorphic functions(see [4]) to the corresponding theory of algebroid functions. The singular direction for $w(z)$ is one important objects studied in the theory of value distribution of algebroid functions. Many people, such as Valiron [14], Lü [7], Lănd Gu [8] and Gao and Wang [2], have studied the Julia direction and Borel direction of algebroid functions.

Recently, Zheng [16] introduced a new singular direction of meromorphic functions, called the $T$ direction. Then Guo, Zheng and $\mathrm{Ng}[3]$ proved that a meromorphic function $f(z)$ must have at least one $T$ direction, provided that $\limsup \left(T(r, f) /(\log r)^{2}\right)=\infty$. Thus a natural question is whether there exists a $T$ direction for an algebroid function $w(z)$. In this paper, we investigate the existence of the $T$ direction dealing with its multiple values for an algebroid function with finite lower order (that is, $\left.\liminf _{r \rightarrow \infty}\left(\left(\log ^{+} T(r, w)\right) / \log r\right)<\infty\right)$, which implies that the algebroid function has a $T$ direction, under the condition $\limsup _{r \rightarrow \infty}\left(T(r, w) /(\log r)^{2}\right)=\infty$.

Let $w(z)$ is a $\nu$-valued algebroid function, defined by (1). Then the single-valued domain $\widetilde{R}_{z}$ of its definition is a Riemann surface, which is a $\nu$-sheet covering of the $z$ plane. A point in $\widetilde{R}_{z}$ is denoted by $\tilde{z}$ if its projection in the $z$-plane is $z$. The open set

Received 21st November, 2006

The project is supported by National Basic Research Program of China(973 Program, Grant No. 2005CB321902).

Copyright Clearance Centre, Inc. Serial-fee code: $0004-9727 / 07 \$ \$ 2.00+0.00$. 
which lies over $|z|<r$ is denoted by $|\bar{z}|<r$, and its boundary is denoted by $|\bar{z}|=r$. Let $n(r, a)$ be the number of $a$-points of $w(z)$ in $|z|<r$, counted according to their multiplicities, and $\bar{n}^{l)}(r, a)$ be the number of distinct $a$-points of $w(z)$ with multiplicity $\leqslant l$ in $|\bar{z}|<r$. In addition, $\bar{n}(r, a)$ denotes the number of distinct $a$-points of $w(z)$ in $|\bar{z}|<r$, ignoring the multiplicity. Let

$$
\begin{aligned}
S(r, w) & =\frac{1}{\pi} \iint_{|z|<r} \frac{\left|w^{\prime}(\tilde{z})\right|^{2}}{\left(1+|w(\tilde{z})|^{2}\right)^{2}} d \omega=\frac{1}{\pi} \sum_{j=1}^{\nu} \iint_{|z|<r} \frac{\left|w_{j}^{\prime}(z)\right|^{2}}{\left(1+\left|w_{j}(z)\right|^{2}\right)^{2}} d x d y, \\
T(r, w) & =\frac{1}{\nu} \int_{0}^{r} \frac{S(r, w)}{t} d t, \quad N(r, a)=\frac{1}{\nu} \int_{0}^{r} \frac{n(t, a)-n(0, a)}{t} d t+\frac{n(0, a)}{\nu} \log r, \\
\bar{N}^{\prime)}(r, a) & =\frac{1}{\nu} \int_{0}^{r} \frac{\left.\bar{n}^{l)}(t, a)-\bar{n}^{l}\right)(0, a)}{t} d t+\frac{\bar{n}^{l}(0, a)}{\nu} \log r, \\
\bar{N}(r, a) & =\frac{1}{\nu} \int_{0}^{r} \frac{\bar{n}(t, a)-\bar{n}(0, a)}{t} d t+\frac{\bar{n}(0, a)}{\nu} \log r, \\
m(r, w) & =\frac{1}{2 \pi \nu} \int_{|\overline{\mid}|=r} \log ^{+}|w(\tilde{z})| d \theta=\frac{1}{\nu} \sum_{j=1}^{\nu} m\left(r, w_{j}\right)=\frac{1}{\nu} \sum_{j=1}^{\nu} \frac{1}{2 \pi} \int_{0}^{2 \pi} \log ^{+}\left|w_{j}\left(r e^{i \theta}\right)\right| d \theta,
\end{aligned}
$$

where $S(r, w)$ is called the mean covering number of $|\bar{z}| \leqslant r$ into the $w$-sphere, and we call $T(r, w)$ the characteristic function of $w(z)$. It is known from [5, pp. 84] that

$$
T(r, w)=m(r, w)+N(r, w)+O(1) .
$$

Let $n\left(r, \widetilde{R}_{z}\right)$ be the number of the branch points of $\widetilde{R}_{z}$ in $|\tilde{z}|<r$, counted with the order of the branch point. Set

$$
N\left(r, \widetilde{R}_{z}\right)=\frac{1}{\nu} \int_{0}^{r} \frac{n\left(t, \widetilde{R}_{z}\right)-n\left(0, \widetilde{R}_{z}\right)}{t} d t+\frac{n\left(0, \widetilde{R}_{z}\right)}{\nu} \log r
$$

From [5], we know that

$$
N\left(r, \widetilde{R}_{z}\right) \leqslant 2(\nu-1) T(r, w)+O(1) .
$$

Next, we define the angle region in the $z$-plane,

$$
\begin{aligned}
& \triangle\left(\theta_{0}, \delta\right)=\left\{z|| \arg z-\theta_{0} \mid<\delta, 0 \leqslant \theta_{0}<2 \pi, 0<\delta<\frac{\pi}{2}\right\}, \\
& \bar{\triangle}\left(\theta_{0}, \delta\right)=\left\{z|| \arg z-\theta_{0} \mid \leqslant \delta\right\} .
\end{aligned}
$$

$\tilde{\Delta}\left(\theta_{0}, \delta\right)$ and $\tilde{\bar{\Delta}}\left(\theta_{0}, \delta\right)$ denote the regions where $\widetilde{R}_{z}$ lies over $\Delta\left(\theta_{0}, \delta\right)$ and $\bar{\Delta}\left(\theta_{0}, \delta\right)$, respectively. Let $n\left(r, \Delta\left(\theta_{0}, \delta\right), a\right)$ be the number of $a$-points of $w(z)$ in $\{|\bar{z}|<r\} \cap \widetilde{\Delta}\left(\theta_{0}, \delta\right)$, counted according to their multiplicities, and $\bar{n}^{r)}\left(r, \Delta\left(\theta_{0}, \delta\right), a\right)$ be the number of distinct $a$-points of $w(z)$ with multiplicity $\leqslant l$ in $\{|\tilde{z}|<r\} \cap \tilde{\Delta}\left(\theta_{0}, \delta\right)$. Then $\bar{n}\left(r, \Delta\left(\theta_{0}, \delta\right), a\right)$ denotes the number of distinct $a$-points of $w(z)$ in $\{|\tilde{z}|<r\} \cap \tilde{\Delta}\left(\theta_{0}, \delta\right)$, ignoring multiplicity. In addition, $n\left(r, \Delta\left(\theta_{0}, \delta\right), \widetilde{R}_{z}\right)$ denotes the number of the branch points in 
$\{|\tilde{z}|<r\} \cap \tilde{\Delta}\left(\theta_{0}, \delta\right)$, counted with the order of branch. Just like the above, we define $N\left(r, \Delta\left(\theta_{0}, \delta\right), a\right), \bar{N}^{l}\left(r, \Delta\left(\theta_{0}, \delta\right), a\right), \bar{N}\left(r, \Delta\left(\theta_{0}, \delta\right), a\right)$ and $N\left(r, \Delta\left(\theta_{0}, \delta\right), \tilde{R}_{z}\right)$, respectively.

DEFINITION 1: Let $w(z)$ be a $\nu$-valued algebroid function defined by (1). The radial $\arg z=\theta_{0}$ is called a $T$ direction of $w(z)$, if for arbitrary $\delta>0(0<\delta<\pi / 2)$, in the angle region $\triangle\left(\theta_{0}, \delta\right)$, given any $a \in \bar{C}$, we have

$$
\limsup _{r \rightarrow \infty} \frac{N\left(r, \Delta\left(\theta_{0}, \delta\right), a\right)}{T(r, w)}>0,
$$

possibly with the exception of at most $2 \nu$ values of $a$.

Definition 2: Let $w(z)$ be a $\nu$-valued algebroid function defined by $(1)$, and $l(>2 \nu)$ be a positive integer. For arbitrary $\delta>0(0<\delta<\pi / 2)$, if

$$
\limsup _{r \rightarrow \infty} \frac{\bar{N}^{l)}\left(r, \Delta\left(\theta_{0}, \delta\right), a\right)}{T(r, w)}>0
$$

holds for any $a \in \bar{C}$ except at most $2 \nu$ possible exceptions, then the radial $\arg z=\theta_{0}$ is called a $T$ direction dealing with multiple value of $w(z)$.

REMARK. From the definitions, it is easy to see that the $T$ direction dealing with multiple value is more precise, and the $T$ direction dealing with multiple values must be a $T$ direction. In addition, if $l=\infty$, (3) implies that

$$
\limsup _{r \rightarrow \infty} \frac{\bar{N}\left(r, \Delta\left(\theta_{0}, \delta\right), a\right)}{T(r, w)}>0 .
$$

THEOREM 1. Let $w(z)$ be a $\nu$-valued algebroid function defined by (1), satisfying

$$
\liminf _{r \rightarrow \infty} \frac{\log ^{+} T(r, w)}{\log r}=\mu<\infty, \quad \underset{r \rightarrow \infty}{\limsup } \frac{T(r, w)}{(\log r)^{2}}=\infty .
$$

Then $w(z)$ must have at least one $T$ direction dealing with multiple values.

\section{LEMMAS}

In order to prove the theorem, we need the following lemmas.

LEMMA 1. ([9]) Let $T(r)$ be a positive and nondecreasing continuous function in $[0, \infty)$, satisfying

$$
\liminf _{r \rightarrow \infty} \frac{\log ^{+} T(r)}{\log r}=\mu<\infty, \quad \underset{r \rightarrow \infty}{\limsup } \frac{T(r)}{(\log r)^{2}}=\infty .
$$

Then for any given $h>0$, there must exist sequences $\left\{r_{n}\right\}$ and $\left\{R_{n}\right\}$ satisfying

$$
R_{n}^{1-o(1)} \leqslant r_{n} \leqslant R_{n}(n \rightarrow \infty),
$$


such that

$$
\lim _{n \rightarrow \infty} \frac{T\left(r_{n}\right)}{\left(\log r_{n}\right)^{2}}=\infty, \quad T\left(e^{h} R_{n}\right) \leqslant e^{h \mu} T\left(R_{n}\right)(1+o(1))(n \rightarrow \infty) .
$$

LEMMA 2. ([2]) Suppose that $w=w(z)(|z| \leqslant R)$ is a $\nu$-valued algebroid function defined by (1), and $a_{1}, a_{2}, \ldots, a_{q}$ are $q(\geqslant 3)$ distinct points in the $w$-sphere such that the spherical distance of any two points is no small than $\delta \in(0,1 / 2)$. Then for any $r \in(0, R)$, we have

$$
\left(q-2-\frac{2}{l}\right) S(r, w) \leqslant \sum_{j=1}^{q} \vec{n}^{l)}\left(R, a_{j}\right)+\frac{l+1}{l} n\left(R, \widetilde{R}_{z}\right)+\frac{C R}{\delta^{10}(R-r)}
$$

where $l \geqslant 3$ is a positive integer and $C$ is a constant.

LEMMA 3. Suppose that $w=w(z)(|z| \leqslant R)$ is a $\nu$-valued algebroid function defined by (1) and $l(>2 \nu)$ is a positive integer. Let $0<\delta<\delta_{0}<\pi / 2,0 \leqslant \theta_{0}<2 \pi$; that is, $\bar{\triangle}\left(\theta_{0}, \delta\right) \subset \Delta\left(\theta_{0}, \delta_{0}\right)$. The part of $\widetilde{R}_{z}$ which lies over $\bar{\Delta}\left(\theta_{0}, \delta\right) \cap\{|z| \leqslant r\}$ is denoted by $\widetilde{\widetilde{\Delta}}\left(\theta_{0}, \delta, r\right)$, and

$$
S\left(r, \bar{\Delta}\left(\theta_{0}, \delta\right), w\right)=\frac{1}{\pi} \iint_{\tilde{\bar{\Delta}}\left(\theta_{0}, \delta, r\right)} \frac{\left|w^{\prime}(\tilde{z})\right|^{2}}{\left(1+|w(\bar{z})|^{2}\right)^{2}} d \omega .
$$

Then for any positive number $\lambda>1$, any positive integer $\alpha$ and any $q(\geqslant 3)$ distinct points $a_{1}, a_{2}, \ldots, a_{q}$ in the $w$-sphere, we have

$$
\begin{aligned}
&(q-2-\left.\frac{2}{l}\right) S\left(r, \bar{\Delta}\left(\theta_{0}, \delta\right), w\right) \\
&\left.\leqslant 2 \sum_{j=1}^{q} \bar{n}^{l}\right)\left(\lambda^{2 \alpha} r, \Delta\left(\theta_{0}, \delta_{0}\right), a_{j}\right)+\frac{l+1}{l}\left(\frac{1+\alpha}{\alpha}\right) n\left(\lambda^{2 \alpha} r, \Delta\left(\theta_{0}, \delta_{0}\right), \widetilde{R}_{z}\right) \\
& \quad+\left(q-2-\frac{2}{l}\right) S\left(\lambda^{\alpha}, \bar{\Delta}\left(\theta_{0}, \delta\right), w\right)+\frac{2 A}{(1-\kappa) \log \lambda} \log ^{+} r
\end{aligned}
$$

where $A$ is a constant depending only on $a_{i}(i=1,2, \ldots, q)$ and $\kappa(0<\kappa<1)$ is a constant depending only on $\delta, \delta_{0}, \lambda$ and $\alpha$.

PROOF: Set $r_{\mu}=\lambda^{\alpha \mu}, r_{\mu, k}=\lambda^{\alpha \mu+k}(\mu=0,1,2, \ldots, 0 \leqslant k \leqslant \alpha-1)$, where $r_{\mu, 0}$ $=r_{\mu}, r_{\mu, \alpha}=r_{\mu+1,0}$. Let $\Omega_{\mu, k}=\left\{z\left|r_{\mu, k} \leqslant\right| z \mid \leqslant r_{\mu, k+1}\right\} \cap \Delta\left(\theta_{0}, \delta_{0}\right)$. For a given positive integer $n$, we have

$$
\sum_{k=0}^{\alpha-1} \sum_{\mu=0}^{n} \Omega_{\mu, k}=\left\{z\left|1=r_{0} \leqslant\right| z \mid \leqslant r_{n+1}\right\} \cap \Delta\left(\theta_{0}, \delta_{0}\right),
$$

for which the component is non-empty. Without loss of generality, we assume that $k=0$ such that

$$
\sum_{\mu=0}^{n} n\left(\Omega_{\mu, 0}, \widetilde{R}_{z}\right) \leqslant \frac{1}{\alpha} n\left(r_{n+1}, \Delta\left(\theta_{0}, \delta_{0}\right), \widetilde{R}_{z}\right),
$$


where $n\left(\Omega_{\mu, 0}, \widetilde{R}_{z}\right)$ denotes the number of branch points in $\widetilde{\Omega}_{\mu, 0}$, counted with the order of the branch point. Let

$$
\begin{aligned}
& \triangle_{\mu}=\left\{z\left|r_{\mu, 0}<\right| z \mid<r_{\mu+1,1}\right\} \cap \Delta\left(\theta_{0}, \delta_{0}\right), \\
& \bar{\triangle}_{\mu}=\left\{z\left|\frac{r_{\mu, 0}+r_{\mu, 1}}{2} \leqslant\right| z \mid \leqslant \frac{r_{\mu+1,0}+r_{\mu+1,1}}{2}\right\} \cap \bar{\Delta}\left(\theta_{0}, \delta\right) .
\end{aligned}
$$

By the Riemann Mapping Theorem, there exists an univalent function $f(z)$ mapping $\triangle_{\mu}$ onto the unit disk $|\zeta|<1$. At the same time, the point

$$
z=\left(\left(r_{\mu, 0}+r_{\mu, 1}+r_{\mu+1}, 0+r_{\mu+1,1}\right) / 4\right) e^{i \theta_{0}}
$$

can be mapped to $\zeta=0$, and $\bar{\Delta}_{\mu}$ can be mapped into $|\zeta| \leqslant \kappa$, where $\kappa(0<\kappa<1)$ is a constant depending only on $\delta, \delta_{0}$ and $\lambda^{a}$, independent of $\nu$. Then from the fact that

$$
S\left(\bar{\Delta}_{\mu}, w\right)=\frac{1}{\pi} \iint_{\overline{\bar{\Delta}}_{\mu}} \frac{\left|w^{\prime}(\widetilde{z})\right|^{2}}{\left(1+|w(\widetilde{z})|^{2}\right)^{2}} d w
$$

is a conformal invariant and Lemma 2, we deduce that

$$
\begin{aligned}
\left(q-2-\frac{2}{l}\right) S\left(\bar{\triangle}_{\mu}, w\right) & \leqslant\left(q-2-\frac{2}{l}\right) S\left(\kappa, w\left(f^{-1}\right)\right) \\
& \left.\leqslant \sum_{j=1}^{q} \bar{n}^{l}\right)\left(|\zeta| \leqslant 1, a_{j}\right)+\frac{l+1}{l} n\left(|\zeta| \leqslant 1, \widetilde{R}_{z}\right)+\frac{A}{1-\kappa} \\
& =\sum_{j=1}^{q} \bar{n}^{l)}\left(\triangle_{\mu}, a_{j}\right)+\frac{l+1}{l} n\left(\triangle_{\mu}, \widetilde{R}_{z}\right)+\frac{A}{1-\kappa} .
\end{aligned}
$$

Thus

(4) $\left(q-2-\frac{2}{l}\right) \sum_{\mu=1}^{n-1} S\left(\bar{\triangle}_{\mu}, w\right) \leqslant \sum_{j=1}^{q} \sum_{\mu=1}^{n-1} \bar{n}^{l)}\left(\triangle_{\mu}, a_{j}\right)+\frac{l+1}{l} \sum_{\mu=1}^{n-1} n\left(\triangle_{\mu}, \tilde{R}_{z}\right)+\frac{n A}{1-\kappa}$.

In addition, it is easy to see that

$$
\sum_{\mu=1}^{n-1} S\left(\bar{\triangle}_{\mu}, w\right) \geqslant S\left(r_{n}, \bar{\Delta}\left(\theta_{0}, \delta\right), w\right)-S\left(r_{1}, \bar{\Delta}\left(\theta_{0}, \delta\right), w\right) .
$$

Since $\left\{\triangle_{\mu}\right\}_{\mu=0}^{n}$ overlays $\left\{\Omega_{\mu, 0}\right\}_{\mu=0}^{n}$ twice at most, we have

$$
\begin{aligned}
& \sum_{\mu=1}^{n-1} \vec{n}^{l}\left(\Delta_{\mu}, a_{j}\right) \leqslant 2 \bar{n}^{l}\left(r_{n+1}, \Delta\left(\theta_{0}, \delta_{0}\right), a_{j}\right), \\
& \sum_{\mu=1}^{n-1} n\left(\Delta_{\mu}, \widetilde{R_{z}}\right) \leqslant\left(1+\frac{1}{\alpha}\right) n\left(r_{n+1}, \Delta\left(\theta_{0}, \delta_{0}\right), \widetilde{R_{z}}\right),
\end{aligned}
$$


where $n\left(\Delta_{\mu}, \widetilde{R}_{z}\right)$ denotes the number of branch points in $\widetilde{\Delta}_{\mu}$, counted with the order of the branch point. And it follows $n=\left(\log r_{n}\right) /(\alpha \log \lambda)$ from $r_{n}=\lambda^{\alpha n}$. Then by (4)-(7), we obtain

(8) $\left(q-2-\frac{2}{l}\right) S\left(r_{n}, \bar{\Delta}\left(\theta_{0}, \delta\right), w\right)$

$$
\begin{aligned}
\leqslant 2 \sum_{j=1}^{q} \bar{n}^{l)}\left(r_{n+1}, \Delta\right. & \left.\left(\theta_{0}, \delta_{0}\right), a_{j}\right)+\frac{l+1}{l}\left(1+\frac{1}{\alpha}\right) n\left(r_{n+1}, \Delta\left(\theta_{0}, \delta_{0}\right), \widetilde{R_{z}}\right) \\
& +\left(q-2-\frac{2}{l}\right) S\left(r_{1}, \bar{\Delta}\left(\theta_{0}, \delta\right), w\right)+\frac{A}{\alpha(1-\kappa) \log \lambda} \log ^{+} r_{n} .
\end{aligned}
$$

If $r \geqslant r_{1}=\lambda^{\alpha}$, there must exist a $n>1$ such that $r_{n-1} \leqslant r<r_{n}$. Thus we have $r_{n+1}=\lambda^{2 \alpha} r_{n-1} \leqslant \lambda^{2 \alpha} r$ and $r_{n} \leqslant \lambda^{\alpha} r \leqslant r^{2}$. Then (8) becomes

$$
\begin{aligned}
& \left(q-2-\frac{2}{l}\right) S\left(r, \bar{\triangle}\left(\theta_{0}, \delta\right), w\right) \\
& \leqslant 2 \sum_{j=1}^{q} \vec{n}^{l)}\left(\lambda^{2 \alpha} r, \Delta\left(\theta_{0}, \delta_{0}\right), a_{j}\right)+\frac{l+1}{l}\left(\frac{1+\alpha}{\alpha}\right) n\left(\lambda^{2 \alpha} r, \Delta\left(\theta_{0}, \delta_{0}\right), \widetilde{R}_{z}\right) \\
& \quad+\left(q-2-\frac{2}{l}\right) S\left(\lambda^{\alpha}, \bar{\Delta}\left(\theta_{0}, \delta\right), w\right)+\frac{2 A}{(1-\kappa) \log \lambda} \log ^{+} r .
\end{aligned}
$$

If $r<r_{1}$, the above inequality is obviously true. The Lemma is complete.

LEMMA 4. Suppose that $w=w(z)(|z| \leqslant R)$, defined by (1), is a $\nu$-valued algebroid function with finite lower order $\mu$, and satisfies

$$
\limsup _{r \rightarrow \infty} \frac{T(r, w)}{(\log r)^{2}}=\infty,
$$

Let $l(>2 \nu), m(>1)$ be both positive integers, and

$$
\eta_{0}=0, \eta_{1}=\frac{2 \pi}{m}, \ldots, \eta_{m-1}=(m-1) \frac{2 \pi}{m}, \eta_{m}=0 .
$$

Then there must exist a $\Delta\left(\eta_{i},(2 \pi) / m\right)$ in $\left\{\Delta\left(\eta_{i},(2 \pi / m)\right)\right\}_{i=0}^{m-1}$ such that

$$
\underset{r \rightarrow \infty}{\limsup } \frac{\vec{N}^{\prime)}\left(r, \Delta\left(\eta_{i},(2 \pi / m)\right), a\right)}{T(r, w)}>0
$$

holds for any $a \in \bar{C}$ except at most $2 \nu$ possible exceptions.

Proof: Suppose that the conclusion is false. Then for any $\Delta\left(\eta_{i},(2 \pi / m)\right)(0 \leqslant i$ $\leqslant m-1)$, there exists $q=2 \nu+1$ exceptional values $\left\{a_{i}^{j}\right\}(j=1, \ldots, 2 \nu+1)$ such that

$$
\limsup _{r \rightarrow \infty} \frac{\bar{N}^{l)}\left(r, \triangle\left(\eta_{i},(2 \pi / m)\right), a_{i}^{j}\right)}{T(r, w)}=0
$$


Thus there exists $R_{0}$, when $r>R_{0}$,

$$
\bar{N}^{l}\left(r, \Delta\left(\eta_{i}, \frac{2 \pi}{m}\right), a_{i}^{j}\right)=o(T(r, w))
$$

holds for $i, j$ uniformly.

Let $\alpha$ is any positive integer. Put $\eta_{i, k}=(2 \pi i / m)+(2 \pi k) /(\alpha m)(0 \leqslant k \leqslant \alpha-1)$. Given any constant $\lambda(>1)$ and $r>R_{0}$, let $\Delta_{i, k}=\left\{z|| z \mid<\lambda^{2 \alpha} r, \eta_{i, k} \leqslant \arg z<\eta_{i, k+1}\right\}$. Then

$$
\sum_{k=0}^{\alpha-1} \sum_{i=0}^{m-1} \triangle_{i, k}=\left\{|z|<\lambda^{2 \alpha} r\right\}
$$

for which the component is non-empty. Without loss of generality, we assume that $k=0$ such that

$$
\sum_{i=0}^{m-1} n\left(\triangle_{i, 0}, \widetilde{R}_{z}\right) \leqslant \frac{1}{\alpha} n\left(\lambda^{2 \alpha} r, \widetilde{R}_{z}\right)
$$

Set

$$
\begin{aligned}
& \bar{\triangle}_{i}=\left\{z \mid\left(\eta_{i, 0}+\eta_{i, 1}\right) / 2 \leqslant \arg z \leqslant\left(\eta_{i+1,0}+\eta_{i+1,1}\right) / 2\right\} \\
& \triangle_{i}=\left\{z \mid \eta_{i, 0}<\arg z<\eta_{i+1,1}\right\} .
\end{aligned}
$$

Since $\left\{\triangle_{i}\right\}_{i=0}^{m-1}$ overlays $\left\{\triangle_{i, 0}\right\}_{i=0}^{m-1}$ only twice, we have

$$
\sum_{i=0}^{m-1} n\left(\lambda^{2 \alpha} r, \triangle_{i, 0}, \widetilde{R}_{z}\right) \leqslant\left(1+\frac{1}{\alpha}\right) n\left(\lambda^{2 \alpha} r, \widetilde{R}_{z}\right)
$$

For $\bar{\triangle}_{i} \subset \triangle_{i}$, applying Lemma 3 , we have

$$
\begin{aligned}
\left(q-2-\frac{2}{l}\right) S\left(r, \bar{\triangle}_{i}, w\right) \leqslant 2 \sum_{j=1}^{q} \bar{n}^{l)}\left(\lambda^{2 \alpha} r, \triangle_{i}, a_{j}\right) & +\frac{l+1}{l}\left(\frac{1+\alpha}{\alpha}\right) n\left(\lambda^{2 \alpha} r, \triangle_{i}, \widetilde{R}_{z}\right) \\
& +\left(q-2-\frac{2}{l}\right) S\left(\lambda^{\alpha}, \bar{\triangle}_{i}, w\right)+\frac{2 A_{i}}{(1-\kappa) \log \lambda} \log ^{+} r
\end{aligned}
$$

Adding from $i=0$ to $m-1$, dividing both sides of this inequality by $r$ and then integrating both sides from 0 to $r$, we obtain the following inequality

$$
\begin{aligned}
\left(q-2-\frac{2}{l}\right) T(r, w) \leqslant 2 \sum_{i=0}^{m-1} \sum_{j=1}^{q} \vec{N}^{l)}\left(\lambda^{2 \alpha} r, \triangle_{i}, a_{j}\right) & +\frac{l+1}{l}\left(\frac{1+\alpha}{\alpha}\right)^{2} N\left(\lambda^{2 \alpha} r, \widetilde{R}_{z}\right) \\
& +\left(q-2-\frac{2}{l}\right) T\left(\lambda^{\alpha}, w\right)+O\left[(\log r)^{2}\right]
\end{aligned}
$$

It follows that by (2) and (9)-(11)

$$
\left(q-2-\frac{2}{l}\right) T(r, w) \leqslant 2(\nu-1) \frac{l+1}{l}\left(\frac{1+\alpha}{\alpha}\right)^{2} T\left(\lambda^{2 \alpha} r, w\right)+o(T(r, w)) .
$$


By Lemma 1 , there exists sequence $\left\{r_{n}\right\}$ and $\left\{R_{n}\right\}$ satisfying $R_{n}^{1-o(1)} \leqslant r_{n} \leqslant R_{n}$ $(n \rightarrow \infty)$, such that

$$
\lim _{n \rightarrow \infty} \frac{T\left(r_{n}, w\right)}{\left(\log r_{n}\right)^{2}}=\infty, \quad T\left(\lambda^{2 \alpha} R_{n}, w\right) \leqslant \lambda^{2 \alpha \mu} T\left(R_{n}, w\right)(1+o(1))(n \rightarrow \infty)
$$

Thus we have

$$
\lim _{n \rightarrow \infty} \frac{T\left(R_{n}, w\right)}{\left(\log R_{n}\right)^{2}}=\infty
$$

Replacing $r$ by $R_{n}$ and dividing both side by $T\left(R_{n}, w\right)$ in (12), we obtain

$$
q-2-\frac{2}{l} \leqslant 2(\nu-1) \frac{l+1}{l}\left(\frac{1+\alpha}{\alpha}\right)^{2} \lambda^{2 \alpha \mu}
$$

when $n \rightarrow \infty$. Letting $\lambda \rightarrow 1, \alpha \rightarrow \infty$, respectively, we deduce $l \leqslant 2 v$ from $q=2 \nu+1$. This contradicts $l>2 \nu$.

\section{Proof of Theorem 1}

By Lemma 4, for any given positive integer $m$, there exists an angle region

$$
\triangle\left(\theta_{m}, \frac{2 \pi}{m}\right)=\left\{z|| \arg z-\theta_{m} \mid<\frac{2 \pi}{m}\right\},
$$

such that

$$
\limsup _{r \rightarrow \infty} \frac{\bar{N}^{l}\left(r, \Delta\left(\theta_{m},(2 \pi / m), a\right)\right.}{T(r, w)}>0
$$

holds for any $a \in \bar{C}$ with at most $2 \nu$ exceptions.

Let $E=\left\{\theta_{m}=\arg z_{m} \mid 0 \leqslant \theta_{m}<2 \pi, m=1,2, \ldots\right\}$, then there exists at least one accumulation point $\theta_{0}\left(0 \leqslant \theta_{0}<2 \pi\right)$ in $E$. Without loss generation, we assume $\theta_{m} \rightarrow \theta_{0}(m \rightarrow \infty)$. Then $J: \arg z=\theta_{0}$ is a $T$ direction dealing with multiple value of $w(z)$. Otherwise there exists a $\delta(0<\delta<\pi / 2)$ and $2 \nu+1$ exceptional values $a \in \Delta\left(\theta_{0}, \delta\right)$, such that

$$
\limsup _{r \rightarrow \infty} \frac{\bar{N}^{l}\left(r, \Delta\left(\theta_{0}, \delta\right), a\right)}{T(r, w)}=0 .
$$

For sufficiently large $m$, we have $\Delta\left(\theta_{m},(2 \pi / m)\right) \subset \Delta\left(\theta_{0}, \delta\right)$. Then

$$
\limsup _{r \rightarrow \infty} \frac{\bar{N}^{l}\left(r, \Delta\left(\theta_{m}, 2 \pi / m\right), a\right)}{T(r, w)}=0
$$

This contradicts the choice of $\left\{\theta_{m}\right\}$. This completes the Theorem.

REMARK 1. If $w(z)$ has finite positive order $\rho$, that is, $\limsup \left(\log ^{+} T(r, w)\right) / \log r$ $=\rho(0<\rho<\infty)$, then its Borel direction of the largest type (see [1]) is also the $T$ direction. Thus there is a sequence filling disks in any $T$ direction (dealing with multiple value)(see $[1,15])$. 
REMARK 2. It remains open, whether the $T$ direction(dealing with multiple value) of $w(z)$ exists, when the lower order of $w(z)$ is infinite; that is,

$$
\liminf _{r \rightarrow \infty} \frac{\log ^{+} T(r, w)}{\log r}=\infty
$$

REMARK 3. If $a(z)$ is a function such that $T(r, a)=o(T(r, w))$, is it true that

$$
\underset{r \rightarrow \infty}{\limsup } \frac{N\left(r, \Delta\left(\theta_{0}, \delta\right), a\right)}{T(r, w)}>0
$$

with the possible exception of at most $2 \nu$ small functions $a(z)$ ?

\section{REFERENCES}

[1] Z.S. Gao and D.S. Sun, 'On the Borel direction of the largest type of algebroid functions', (in Chinese), Chinese Ann. Math. Ser.A 6 (1997), 701-710.

[2] Z.S. Gao and F.Z. Wang, 'Theorems of the covering surfaces and multiple values of the algebroid functions', (in Chinese), Acta. Math. Sinica. 44 (2001), 805-814.

[3] H. Guo, J.H. Zheng and T. Ng, 'On a new singular direction of meromorphic functions', Bull. Austral. Math. Soc. 69 (2004), 277-287.

[4] W.K. Hayman, Meromorphic functions (Clarendon Press, Oxford, 1964).

[5] Y.Z. He amd X.Z. Xiao, Algebroid functions and ordinary differential equations, (in Chinese) (Science Press, Beijing, 1988).

[6] K. Katajamäki, 'Algebroid solutions of binomial and linear differential equations', Ann. Acad. Sci. Fenn. Ser. A I Math. 90 (1993).

[7] Y.N. Lü, 'On the Julia direction of meromprphic functions and meromorphic algebroid functions', (in Chinese), Acta Math. Sinica 27 (1984), 368-373.

[8] Y.N. Lü and Y.X. Gu, 'On the existence of Borel direction for algebroid function', (in Chinese), Chinese Sci. Bull. 28 (1983), 264-266.

[9] Y.N. Lü and G.H. Zhang, 'On the Nevanlinna direction of meromorphic functions', Sci. China Ser. A. 3 (1983), 215-224.

[10] H.L. Selberg, 'Über eine Eigenschaft der logarithmischen Ableitung einer meromorphen oder algebroiden Funktion endlicher Ordnung', Avh. Norske Vid. Akad. Oslo I 14 (1929).

[11] H.L. Selberg, 'Über die Wertverteilung der algebroiden funktionen', Math. Z. 31 (1930), 709-728.

[12] E. Ullrich, 'Über den Einfluss der Verzweigtheit einer albebroide auf ihre Wertverteilung', J. Reine Angew. Math. 167 (1931), 198-220.

[13] G. Valiron, 'Sur les fonctions algébroïdes méromorphes du second degré', C. R. Acad. Sci. Paris. 189 (1929), 623-625.

[14] G. Valiron, 'Sur les directions de Borel des fonctions algébrodes méromorphes d'ordre infini', C. R. Acad. Sci. Paris 206 (1938), 735-737.

[15] Z.X. Xuan and Z.S. Gao, 'The Borel direction of the largest type of algebroid functions dealing with multiple values', Kodai Math. J. 30 (2007), 97-110. 
[16] J.H. Zheng, 'On transcendental meromorphic functions with radially distributed values', Sci. China Ser. A. 47 (2004), 401-416.

LMIB \& Department of Mathematics

Beihang University

Beijing, 100083

People's Republic of China

e-mail: wsmin@ss.buaa.edu.cn
Department of Mathematics

Beihang University

Beijing, 100083

People's Republic of China

e-mail: zshgao@buaa.edu.cn 